\title{
Editorial
}

\section{Cross-Layer Optimized Wireless Multimedia Communications}

\author{
Zhu Han, ${ }^{1}$ Haohong Wang, ${ }^{2}$ D. Oliver Wu, ${ }^{3}$ Jianwei Huang, ${ }^{4}$ and M. Van Der Schaar ${ }^{5}$ \\ ${ }^{1}$ Department of Electrical and Computer Engineering, Boise State University, 1910 University Drive, Boise, ID 83725, USA \\ ${ }^{2}$ Marvell Semiconductor Incorporation, 5488 Marvell Lane, Santa Clara, CA 95054, USA \\ ${ }^{3}$ Department of Electrical and Computer Engineering, University of Florida, P.O.Box 116130, Gainesville, FL 32611-6130, USA \\ ${ }^{4}$ Department of Information Engineering, The Chinese University of Hong Kong, Shatin, NT, Hong Kong SAR, China \\ ${ }^{5}$ Electrical Engineering Department, University of California Los Angeles (UCLA), 66-147E Engineering IV Building, \\ 420 Westwood Plaza, Los Angeles, CA 90095-1594, USA
}

Received 26 August 2007; Accepted 26 August 2007

Copyright (c) 2007 Zhu Han et al. This is an open access article distributed under the Creative Commons Attribution License, which permits unrestricted use, distribution, and reproduction in any medium, provided the original work is properly cited.

Recent advances in wireless and mobile communications provide ample opportunities for introducing new services. Supporting multimedia applications and services over wireless networks is challenging due to constraints and heterogeneities such as limited battery power, limited bandwidth, random time-varying fading effect, different protocols and standards, and stringent quality-of-service (QoS) requirements. Cross-layer design methodologies provide great promises for addressing these challenges and achieving reliable and high-quality end-to-end performance in wireless multimedia communications. In this special issue on crosslayer wireless multimedia communications, we have accepted a few papers that address such issues.

The first paper, "Quality-based backlight optimization for video playback on handheld devices," considers the problem caused by limited power consumption supply of mobile devices and the intensive battery resource request of the multimedia streaming applications. In this work, effective techniques for backlight energy saving are proposed to optimize the backlight dimming while maintaining acceptable service quality.

Both the second paper, "Asymptotic delay analysis for cross-layer delay-based routing in ad hoc networks," and the third paper, "A study on the usage of cross-layer power control and forward error correction for embedded video transmission over wireless links," provide analytical framework and evaluations for media delivery over wireless network. The former work considers multihop networks, where the accurate delay distribution estimation is used for QoS-based routing in multimedia applications. The latter paper considers single-hop network, where the source coding parameters, (e.g., packetization schemes and packet classification), medium access control procedures, (e.g., ARQ and forward error correction), and physical parameters (e.g., transmission power and channel sensing) are jointly optimized.

The forth paper, "Cross-layer path configuration for energy-efficient communication over wireless ad hoc networks," studies the energy-efficient configuration of multihop paths with ARQ mechanism in wireless ad hoc networks. The proposed approach jointly optimize the schedualing of the transmitting power of each transmitted node and the retransmission limit over each hop, under the constraints on maximal delay and minimal packet delivery ratio.

The fifth paper, "MOS-based multiuser multiapplication cross-layer optimization for mobile multimedia communication," proposes a cross-layer optimization strategy that jointly optimizes the application layer, the data-link layer, and the physical layer using a novel optimization scheme based on the mean opinion score as the unifying metric over different application classes.

The sixth paper, "Cross-layer perceptual ARQ for video communications over 802.11e wireless networks," presents an application-level perceptual ARQ algorithm for video streaming over 802.11e wireless networks. A simple and effective formula is proposed to combine the perceptual and temporal importance of each packet into a single priority value.

The seventh paper, "Cross-layer design of source rate control and congestion control for wireless video streaming," extends the QoS-aware congestion control mechanism to the wireless scenario, and provides a detailed discussion about how to enhance the overall performance in terms of rate smoothness and responsiveness of the transport protocol.

The eighth paper, "Identifying opportunities for exploiting cross-layer interactions in adaptive wireless systems," 
presents an analysis of the interaction of physical data link and network layer parameters with respect to throughput, bit-error rate, delay, and jitter. The goal of this analysis is to identify opportunities where systems designers might exploit cross-layer interactions to improve the performance of voice over IP, instant messaging, and file transfer applications.

The ninth paper, "MAC-layer QoS management for stream-ing rate-adaptive VBR video over IEEE 802.11e HCCA WLANs," proposes a cross-layer framework for efficiently delivering multiclass rate-adaptive variable bitrate video over HCCA. The proposed framework consists of three major modules: the MAC-layer admission control, the MAClayer resource allocation, and the application-layer video adaptation.

The tenth paper, "A cross-layer optimization approach for energy efficient wireless sensor networks: coalition-aided data aggregation, cooperative communication, and energy balancing," proposes a cross-layer optimization approach to study energy-efficient data transport in coalition-based wireless sensor networks, where neighboring nodes are organized into groups to form coalitions, and sensor nodes within one coalition carry out cooperative communications.

In the final paper of this special issue, "Location-aware cross-layer design using overlay watermarks," location information of a mobile for efficient routing can be easily derived when a unique watermark is associated with each individual transceiver. In addition, cross-layer signaling and other interlayer interactive information can be exchanged with a new data pipe created by modulating the overlay watermarks.

The upcoming new applications for wireless multimedia communications open new opportunities for many interesting and comprehensive research topics targeting at concepts, methodologies, and techniques to support the future advanced mobile wireless applications. Clearly, the developments of the new schemes, mechanisms, and systems associated with the cross-layer designs and protocols will have a significant impact on the next generation of wireless communications and networks.

Zhu Han

Haohong Wang Jianwei Huang M. Van Der Schaar 

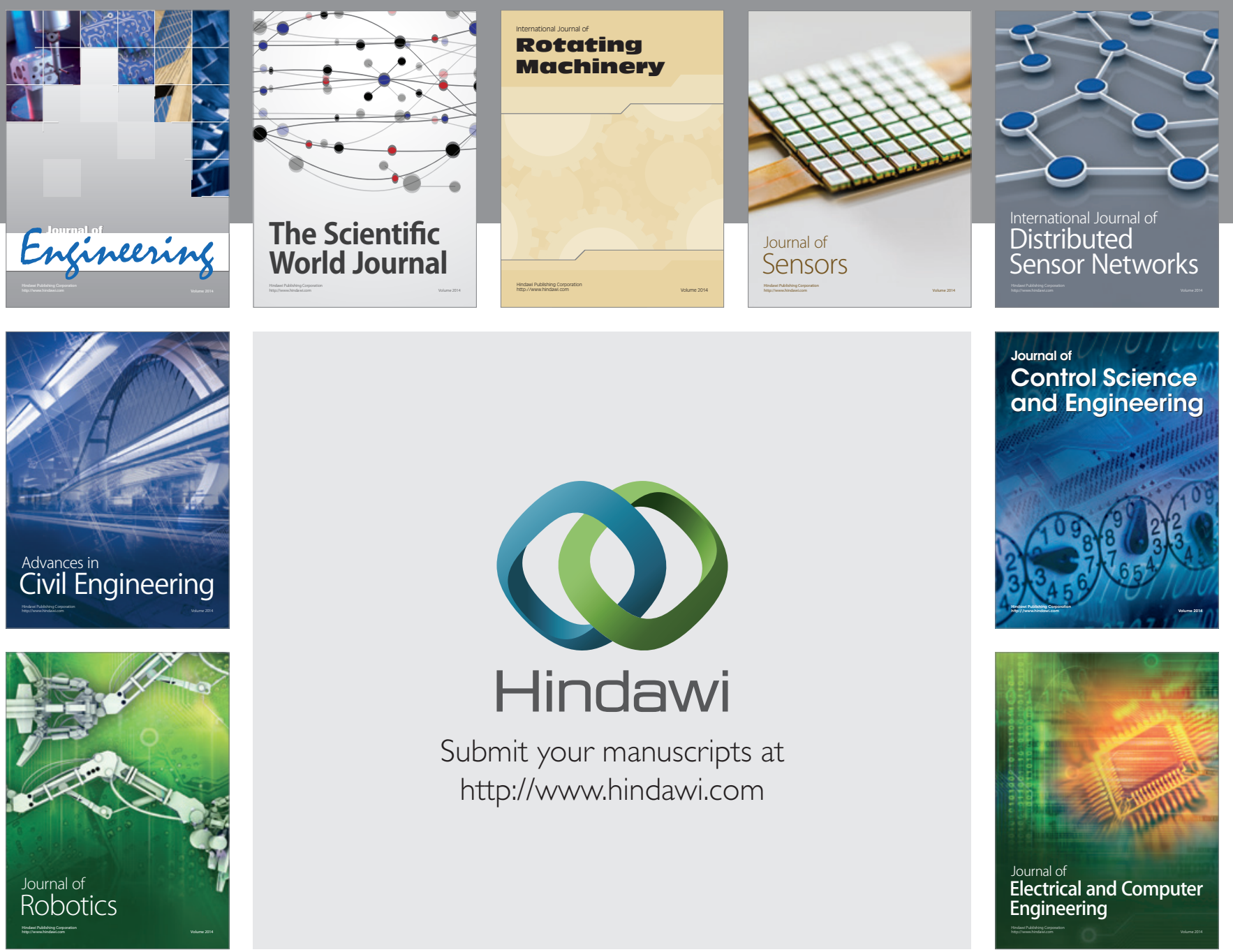

Submit your manuscripts at

http://www.hindawi.com
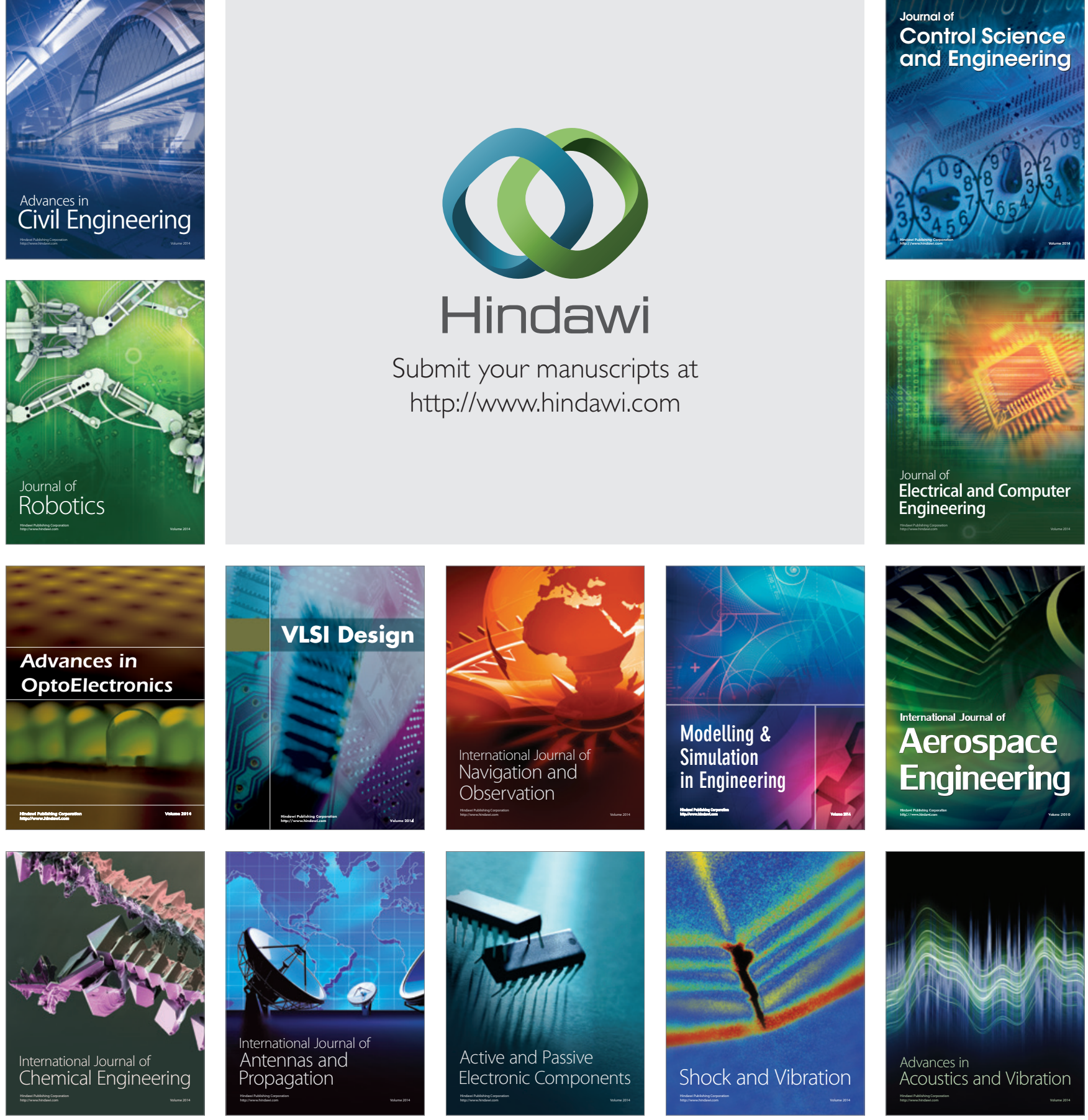